\title{
THE MECHANICAL PROPERTIES OF HUMAN TIBIAL TRABECULAR BONE AS A FUNCTION OF METAPHYSEAL LOCATION
}

\author{
Steven A. Goldstein, Douglas L. Wilson, David A. Sonstegard and \\ LARRY S. MatThews \\ Biomechanics, Trauma and Sports Medicine Laboratory, Department of Surgery, Section of Orthopaedic \\ Surgery, University of Michigan, Ann Arbor, MI, U.S.A.
}

\begin{abstract}
Experimental determination of the elastic modulus and ultimate strength of human tibial trabecular bone as a function of metaphyseal location is presented.

A $1 \mathrm{~cm}$ cubic matrix with planes parallel to the subchondral plate was defined on five fresh frozen cadaver tibias. Approximately $400,7 \mathrm{~mm} \times 10 \mathrm{~mm}$ cylindrical bone plugs were cut from the locations defined by the matrix and tested in uniaxial compressive stress at a strain rate of $0.1 \% \mathrm{~s}^{-1}$. Results of the study indicate that the trabecular bone properties vary as much as two orders of magnitude from one location to another. As might be predicted from Wolff's law, and noted by previous investigators, concentrations of strength arise from the medial and lateral metaphyseal cortices toward the major medial and lateral contact regions.

These results may be valuable for improved analytical modeling and optimal prosthetic design.
\end{abstract}

\section{INTRODUCTION}

Characterization of metaphyseal trabecular bone at the knee joint using various analytical techniques has been an area of great research interest in recent years (Bartel et al., 1982; Hayes et al., 1978; Hayes et al., 1979; Lewis et al., 1982; Murase et al., 1983; Pugh et al., 1973a; Simon, 1980; Williams and Lewis, 1979). Most studies have focused primarily on the load distribution properties relating specifically to improving prosthetic design and selection of optimal and implantable materials.

The function of trabecular bone in human synovial joints is to distribute the large contact stresses applied at the articular surfaces to the compact bone of the cortical shafts (Hayes et al., 1978). It is generally accepted that this function directly influences the structure and strength of the metaphyseal bone; a relationship known as Wolff's law (Wolff, 1870, 1892). Hayes and Snyder (1981) in a recent investigation of Wolff's law have provided strong support for the trajectorial theory of bone architecture. This theory states that the trabeculae are organized along the lines of principal stress (Meyer, 1867; Koch, 1917; Pauwels, 1948; Kummer, 1966).

The mechanical properties of trabecular bone have been shown to be similar to that of fluid filled porous engineering materials (Carter and Hayes, 1977). Its porosity and structural orientation contribute to its anisotropy (Galante et al., 1970; Harrigan et al., 1980; Townsend et al., 1975; Williams and Lewis, 1982). The strength and stiffness of trabecular bone is proportional to its mineral density and orientation (Behrens et al., 1974; Galante et al., 1970; Lindahl, 1976;

Received 15 April 1982; in revised form 11 May 1983.
McElhaney et al., 1970; Pugh et al., 1973b; Schoerfeld et al., 1974; Townsend et al., 1975). Carter and Hayes (1977) found the compressive strength to be related to the square of the apparent density and the compressive modulus to be proportional to the cube of the density. Carter et al. (1980) found similar relationships when the trabecular bone was subjected to pure tension. This relationship between the mechanical properties of trabecular bone and its mineral density is fundamental to recent investigations of noninvasive techniques of property determinations such as computed tomography (Goldstein et al., 1982).

Brown and Ferguson (1980) experimentally determined the spatial and directional variation of the trabecular bone material properties in the proximal femur. Their results indicated that the contour of property variations correlated well with the corresponding $\mathrm{X}$-ray findings. They also concluded that the stiffness and yield point at a given location were linearly proportioned to one another regardless of the degree of anisotropy.

Behrens et al. (1974) determined the properties of trabecular bone at the knee joint. Their study, which evaluated trabecular bone specimens from different contact area locations, suggested that bone strength correlates not only with magnitude but also with duration and frequency of stress normally applied to the area

This study was designed to determine the variation of trabecular bone mechanical properties throughout the entire human tibial metaphysis. This information will provide the material property parameters necessary for more accurate modeling of the tibial metaphysis. The results may therefore be of value for design optimization of total knee prosthesis tibial components. 


\section{MATERIALS AND METHODS}

\section{Bone specimens}

Symmetric cylindrical plugs of trabecular bone with a cross sectional diameter of $7 \mathrm{~mm}$ and a height of $10 \mathrm{~mm}$ were removed from standardized locations in five human tibial metaphyses. The tibias were obtained from five autopsy subjects (three males, two females) between the ages of 50 and 70 and immediately stored at $-20^{\circ} \mathrm{C}$. None of the specimens exhibited severe arthritic pathology, or pathological osteopenia on gross examination. All subjects appeared to be of average weight and stature.

To standardize the plug locations across the specimen population, a coordinate axis with respect to the first plane of trabecular bone beneath the subchondral plate was defined (Fig. 1). This trabecular bone plane was located from three specific points through the articular surface. Two points corresponded to the centroids of the ellipses formed by the semi-lunar cartilages, and a third at the posterior tibial spine (Fig. 1). Three holes were ground through the subchondral bone until trabecular bone was exposed under each point. The tibia was placed in an alignment apparatus with markers matching the three exposed trabecular bone areas. The bone was encased in a polyurethane foam that rigidly fixed the tibia relative to the molding enclosure. This methodology provided standard orientation based on the geometry of each specimen: specifically the location of the approximate centers of the load bearing areas were consistent for all tibias. These defined center points of the load bearing areas corresponded to the centroids of the included ellipses formed by the menisci.

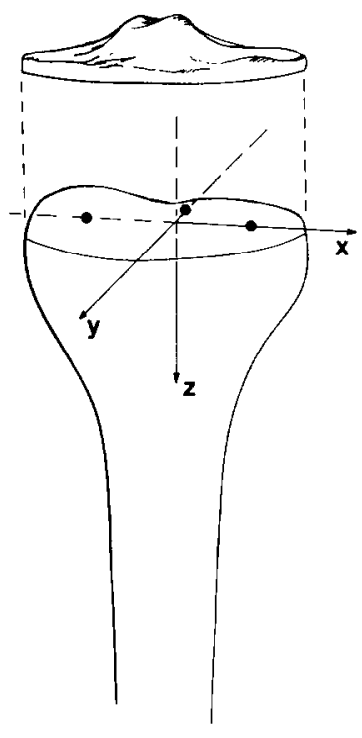

Fig. 1. The first plane of trabecular bone was defined from three points: two corresponding to the centroids of the ellipses formed by the semi-lunar cartilages and a third at the interspinous region. The points were located by drilling through the articular surface and used to reference the first transverse slice.
The encased tibias were then sectioned transversely at $10 \mathrm{~mm}$ intervals with a specially adapted industrial band saw while being constantly cooled with Ringer's solution. The ends of the specimens were carefully machined using a high speed mill to ensure that they were plano-parallel. Each section was then placed in a plexiglass drilling template that defined the locations that the plugs were to be taken. The cylindrical plugs were cut from each section using a trephine bone cutter, and placed in Ringer's solution until testing. Each plug was labeled according to the specific matrix location.

Each specimen was tested with twenty minutes after placement in the Ringer's solution. All the specimens were maintained at constant room temperature until and during testing. Care was taken to expose all specimens to the same environmental conditions, such as time from thawing until testing.

\section{Materials properties}

The plugs were tested in uniaxial compressive stress on an Instron Materials testing machine at a strain rate of $0.1 \% \mathrm{~s}^{-1}$. Simultaneous recordings of load vs deformation were made. Each test specimen was preloaded at 1.3 MPa, returned to zero and then loaded to failure. From the load deformation curves, ultimate load and tangent elastic modulus (at $67 \%$ of ultimate load) were documented. In a very limited number of specimens there was evidence of yielding during preload. In these cases, the preload was extended until failure and the ensuing curves analyzed for tangent modulus and ultimate strength.

\section{RESULTS}

The results of the study indicate the presence of concentrations of strength arising from the medial and lateral metaphyseal cortices and extending inward beneath the contact regions of the femoral condyles. It was also observed that a central column of extremely low modulus trabecular bone extends upward from the intramedullary space towards the subchondral plate. The results indicated that the medial side was stronger and stiffer than the lateral and the lateral side seemed to have a concentration of strength in the vicinity of the attachment of the fibular head.

These patterns of variation were found to be consistent among our specimen population. The mean values of tangent elastic moduli as a function of location are illustrated in Fig. 2. While the mean results illustrate a variation of greater than one order of magnitude in some areas, the individual tibias showed as much as two orders of magnitude differential. A transverse section of one subject illustrates a variation of elastic modulus from $430 \mathrm{MPa}$ in the load bearing areas to $4.2 \mathrm{MPa}$ in the center (Fig. 3). The mean elastic moduli and their standard deviations for the first plane of trabecular bone are illustrated in Table 1. 

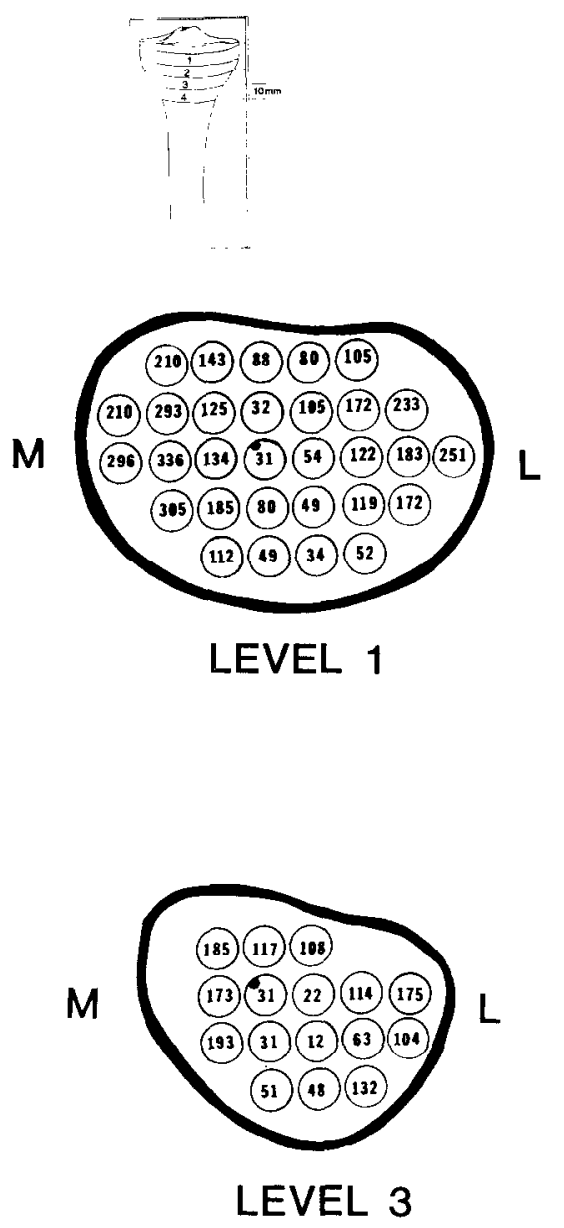
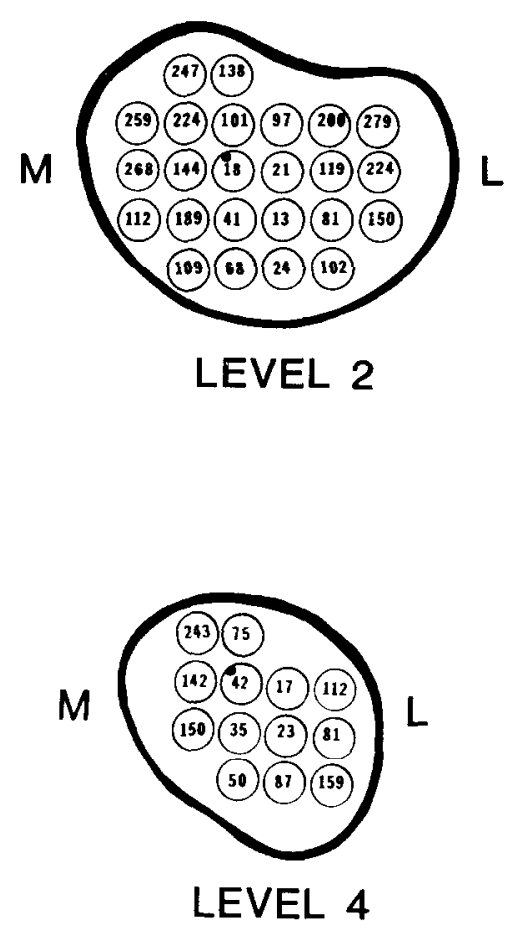

Fig. 2. The elastic moduli (MPa) as a function of location are presented in this figure. These values are the mean responses for the sample population for each plug location.

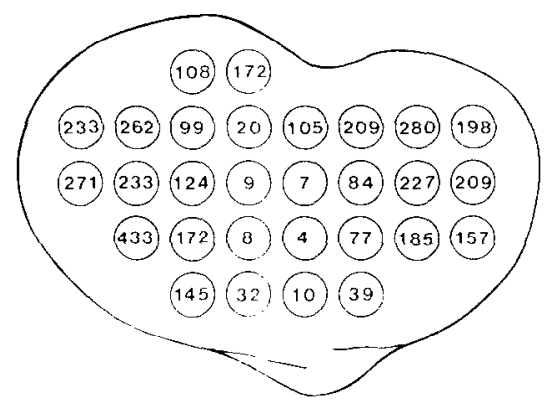

Fig. 3. This figure represents the elastic moduli (MPa) from the first transverse section of one tibia. This individual illustrates a variation in properties of two orders of magnitude.

The ultimate strength of the bone specimens was found to be linearly proportional to the elastic modulus and demonstrated the same patterns of variability. A least squared error regression analysis indicated that this relationship was significant at $p<0.001$ with $R^{2}=0.89$ (Fig. 4). The ultimate strength can be predicted from the elastic modulus by the following model

Ultimate Stress - (0.0265) (Tangent Elastic Modulus).

\section{DISCUSSION}

The results of this study support the hypothesis of bone functional remodeling. Strengthening and stiffening of the bone is clearly evident directly beneath the centers of the load bearing areas. These areas were assumed in this study to be the regions corresponding to the entire surfaces within the outer perimeter of the semilunar cartilages (Kettlekamp and Jacobs, 1972; Walker and Hajek, 1972; Seedholm et al., 1974).

The patterns of increased stiffness become more peripheral, distally in the metaphysis. While this finding supports the hypothesis that the loads are effectively transmitted to the distal cortices, we were unable to obtain adequate samples adjacent to the cortices to substantiate this hypothesis. In a similar nondestructive study, utilizing computer axial tomography, however, this finding was further supported 
Table 1. To illustrate the magnitude of the variation in the experimental data, the mean elastic moduli and standard deviations for the trabecular bone in Level 1 are presented

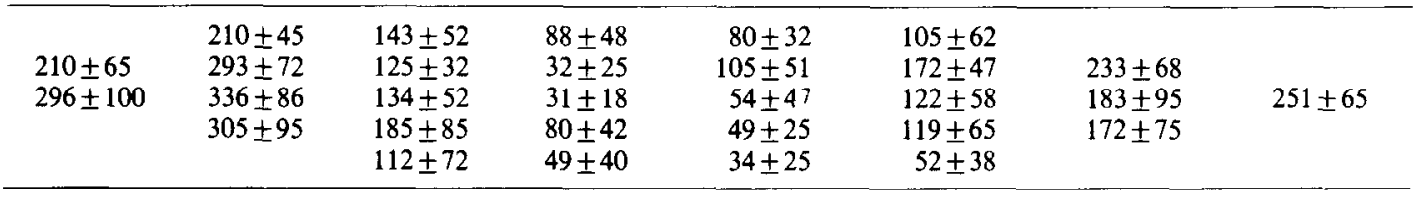

(Mean Tangent Elastic Moduli \pm S.D.)

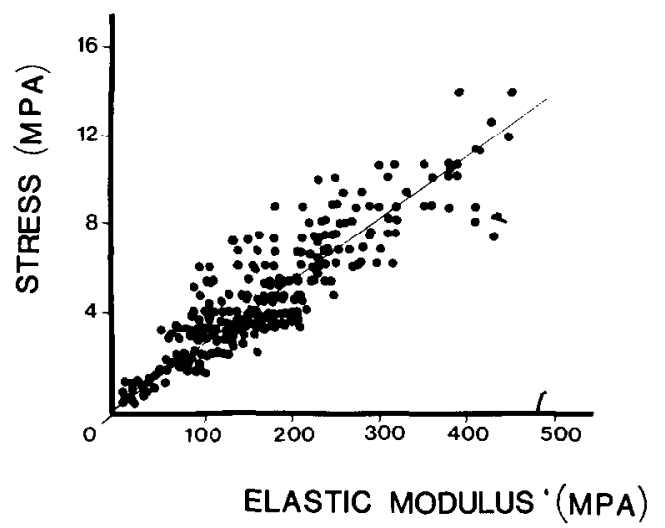

Fig. 4. A statistically significant relationship between elastic modulus and ultimate strength was found.

by an observation of increasing peripheral trabecular density at the more distal metaphyseal regions (Goldstein et al., 1982).

The variation of properties in the first transverse plane is similar to that reported by Behrens et al. (1974). Similarly, the observation of medial side strengthening was also in agreement with observations from other investigators (Behrens et al., 1974; Walker and Hajek, 1972). The range of material properties in the present study were also found to be in general agreement with previous published data on tibial trabecular bone (McElhaney et al., 1970; Pugh et al., 1973b; Carter and Hayes, 1977; Evans and King, 1961; Evans, 1969; Behrens et al., 1974; Hayes and Carter, 1976; Carter and Hayes, 1977).

The linear relationship between the ultimate strength and the elastic modulus was also found to be similar to that previously published by Carter and Hayes (1977).

There are a number of limitations to this information which must be emphasized. Trabecular bone is a difficult material to characterize analytically or experimentally. It is porous or perhaps more accurately it consists of a matrix of plates and columns naturally filled with a fatty viscous fluid. It has been demonstrated that trabecular bone has preferred orientations, so that the macroscopic properties are not isotropic. The structure of trabecular bone is also fairly coarse when compared to the size of the plugs obtained in our study. This causes additional problems, in that any test sample large enough to behave as a continuous material during testing is likely to have a large variation of properties within the sample boundaries. Trabecular bone has been shown to be strain rate dependent as well as anisotropic (Ducheyne et al., 1977; Galante et al., 1970; Harrigan et al., 1980; Townsend et al., 1975; Williams and Lewis, 1982).

While the study does not completely characterize trabecular bone, it does provide some valuable information about the relative variation of modulus and strength in tibias from a typical arthroplasty population. This information may be useful in developing improved models of the tibia and thus improving future prosthetic designs and methods of fixation.

\section{REFERENCES}

Bartel, D. L., Burstein, A. H., Santavicca, E. A. and Insall, J. N. (1982) Performance of the tibial component in total knee replacement. J. Bone Jt Surg. 64A, 1026-1033.

Behrens, J. C., Walker, P. S. and Shoji, H. (1974) Variations in strength and structure of cancellous bone at the knee. J. Biomechanics 7, 201-207.

Brown, T. D. and Ferguson, A. B. (1980) Mechanical property distributions in the cancellous bone of the human proximal femur. Acta orthop. scand. 51, 429-437.

Carter, D. R. and Hayes, W. C. (1976) Bone compressive strength: the influence of density and strain rate. Science 194, 1174-1176.

Carter, D. R. and Hayes, W. C. (1977) The compressive behavior of bone as a two phase porous structure. J. Bone Jt Surg. 59A, 954-967.

Carter, D. R., Schwab, G. H. and Spengler, D. M. (1980) Tensile fracture of cancellous bone. Acta orthop. scand. 51, 733-741.

Ducheyne, P., Heymans, L., Martens, M., Aernoudt, E., deMeester, P. and Mulier, J. D. (1977) The mechanical behavior of intracondylar cancellous bone of the femur at different loading rates. J. Biomechanics 10, 747-762.

Evans, F. G. and King, A. I. (1961) Regional differences in some physical properties of human spongy bone. Biomechanical Studies of the Musculoskeletal System, (Edited by Evans, F. G.). Charles C. Thomas, Springfield, IL.

Evans, F. G. (1969) The mechanical properties of bone. Artif. Limbs 13, 37-48.

Galante, J., Rostoker, W. and Ray, R. D. (1970) Physical properties of trabecular bone. Calcif Tissue Res. 5, 236-246.

Goldstein, S. A., Matthews, L. S., Braunstein, E. M. and Marks, B. (1982) The distribution of tibial trabecular bone as determined by computed axial tomography. Transactions of the Orthopaedic Research Society, New Orleans, Louisiana. ORS, Chicago. 
Harrigan, T. P., Oh, I., Harris, W. H. and Mann, R. W. (1980) The anisotropic properties of trabecular bone. Transactions of 26th Orthopaedic Research Society Vol. 5, p. 60. ORS, Chicago.

Hayes, W. C. and Carter, D. R. (1976) Postyield behavior of subchondral trabecular bone. J. biomed. Mat. Res. Symp. 7, $537-544$.

Hayes, W. C., Swenson, L. W. and Schurman, D. J. (1978) Axisymmetric finite element analysis of the lateral tibial plateau. J. Biomechanics 11, 21-33.

Hayes. W. C. and Snyder, B. (1981) Toward a quantitative formulation of Wolff's law in trabecular bone in mechanical properties of bone. A.S.M.E. Symp. A.M.D. 45, 43-68.

Hayes, W. C. and Snyder, B. (1979) Correlations between stress and morphology in trabecular bone of the patella. Transactions of the 25th Orthopaedic Research Society, Vol. 4, p. 88. ORS, Chicago

Kettlekamp, D. B. and Jacobs, A. W. (1972) Tibiofemoral contact area-determination and implications. J. Bone $J t$ Surg. 54A, 349-356.

Koch, J. C. (1917) The laws of bone architecture. Am. J. Anat. 21, 177-298.

Kummer, B. (1966) Photoelastic studies on the functional structure of bone. Folia biotheor. 6, 31-40.

Lewis, J. L., Askew, M. J. and Jaycox, D. P. (1982) A comparative evaluation of tibial component designs of total knee prostheses. J. Bone Jt Surg. 64A, 129-135.

Lewis. J. L. (1977) Analytical methods in prosthesis design. Proceedings of the Workshop on Internal Joint Replacement. Northwestern University Press, Chicago.

Lindahl, O. (1976) Mechanical properties of dried defatted spongy bone. Acta orthop. scand. 47, 11-19.

McElhaney, H. H., Alem, N. M. and Roberts, V. L. (1970) A porous block model for cancellous bone. Publication no. 70-WA BHF-2, American Society of Mechanical Engineers, New York.

Meyer, H. (1867) Die Architektur der Spongiosa. Arch. Anat. Physiol. 47, 615-628.
Murase, K., Crowninshield, R. D., Peterson, D. R. and Chang, T. S. (1983) An analysis of tibial component design in total knee arthroplasty. J. Biomechanics 16, 13-22.

Pauwels, F. (1948) Bedentung and Kausale Erklung der Spongiosaarchitektur in neuer Auffassung. Arztl. Wschr. 3, 379.

Pugh, J. W., Rose, R. M. and Radin, E. L. (1973a) A structural model for the mechanical behavior of trabecular bone. J. Biomechanics 6, 657-670.

Pugh, J. W., Rose, R. M. and Radin, E. L. (1973b) Elastic and viscoelastic properties of trabecular bone: dependence on structure. J. Biomechanics, 6, 475-486.

Schoenfeld, C. M., Lautenschlager, E. P. and Meyer, P. R. (1974) Mechanical properties of human cancellous bone in the femoral head. Med. Biol. Engng 12, 313-317.

Seedholm, B. B., Dowson, D. and Wright, V. (1974) The load bearing function of the menisci: a preliminary study. The Knee Joint, (Edited by Ingwerson, O. S.). Elsevier, New York.

Simon, B. R. (1980) Finite Elements in Biomechanics. University of Arizona Press, Tucson.

Townsend, P. R., Raux, P., Rose, R. M., Miegel, R. E. and Radin, E. L. (1975) The distribution and anisotropy of the stiffness of cancellous bone in the human patella. $J$. Biomechanics 8, 363-367.

Walker, P. S. and Hajek, J. V. (1972) Load bearing area in the knee joint. J. Biomechanics, 5, 581-589.

Williams, J. L. and Lewis, J. L. (1979) An anisotropic model of cancellous bone. A.S.M.E. 1979 Biomechanics Simposium A.M.D., Vol. 32 .

Williams, J. L. and Lewis, J. L. (1982) Properties and an anisotropic model of cancellous bone from the proximal tibial epiphysis. J. biomech. Engng 104, 50-56.

Wolff, J. (1870) Uber die innere Architektur der Knochen and ihre Bedeutung fur die Frame von Knockenwaschstum. Virchows Arch. path. Anat. Physiol. 50.

Wolff, J. (1892) Pas Destez der Transformation der Knochen. Hirschwald, Berlin. 